\title{
Moara: Flexible and Scalable Group-Based Querying System
}

\author{
Steven Y. Ko ${ }^{1}$, Praveen Yalagandula ${ }^{2}$, Indranil Gupta ${ }^{1}$, \\ Vanish Talwar ${ }^{2}$, Dejan Milojicic ${ }^{2}$, and Subu Iyer ${ }^{2}$ \\ ${ }^{1}$ University of Illinois at Urbana-Champaign \\ ${ }^{2}$ HP Labs, Palo Alto
}

\begin{abstract}
Users and administrators of large-scale infrastructures (e.g., datacenters and PlanetLab) are frequently in need of monitoring groups of machines in the infrastructure. Though there exist several distributed querying systems for this monitoring purpose, they are not group-based; they mostly focus on querying the entire system. In this paper, we present Moara, a new querying system that makes two novel contributions. First, Moara builds aggregation trees for different groups and adaptively maintains the trees to optimize the total message cost. Second, Moara supports a query language allowing groups to be specified implicitly via predicates consisting of arbitrarily nested unions and intersections. Our evaluations on Emulab, on PlanetLab, and with large-scale simulations, demonstrate Moara's ability to answer complex queries within a fraction of a second, to deal with high levels of dynamism in groups, and to incur a low bandwidth overhead per host per query in comparison to existing centralized and distributed aggregation systems.
\end{abstract}

\section{Introduction}

Large-scale distributed infrastructures have become increasingly common in various domains. Today's enterprise data centers [1] are equipped with thousands of machines and run thousands of different applications and services. Federated computing infrastructures such as PlanetLab [2], proposed GENI infrastructure [3], and computational grids [4] consist of thousands of hosts providing resources for a number of projects.

A frequent need of the users and the administrators of such infrastructures is monitoring and querying the status of groups of machines in the infrastructure, as well as the infrastructure as a whole. These groups may be static or dynamic, e.g., the PlanetLab slices, the machines running a particular service in a datacenter, or the machines with CPU utilization above 50\%. Further, users typically desire to express complex criteria for the selection of the host groups to be queried. For example, "find top-3 loaded hosts where (ServiceX $=$ true) and (Apache $=$ true)" is a query that targets two groups - hosts that run service $\mathrm{X}$ and hosts that run Apache. Dynamic groups mean that the size and composition of groups vary across different queries as well as time.

In general, users and administrators desire to monitor the performance of these groups, to troubleshoot any failures or performance degradations, and to track usage of allocated resources. These requirements point to the need for a group-based querying system that can provide instantaneous answers to queries over in-situ data targeting 
one or more groups. In fact, several existing distributed aggregation systems [5, 6, 7] can be considered as a special case of group-based querying systems, as they target querying of only a single group, i.e., the entire system.

Any group-based querying system should satisfy three requirements: flexibility, efficiency, and scalability. First, the system should be flexible to support expressive queries that deal with multiple groups, such as unions and intersections of different groups. Second, the system should be efficient in query resolution-it should minimize the message overhead while responding quickly with an answer. Third, the system should scale with the number of machines, the number of groups, and the rate of queries.

In this paper, we propose Moara, a new group-based distributed aggregation system that targets all three requirements. A query in Moara has three parts: (queryattribute, aggregation function, group-predicate), e.g., (Mem-Util, Average, Apache = true). Moara returns the resulting value from applying the aggregation function over the values of query-attribute at the machines that satisfy the group-predicate.

Moara makes two novel design contributions over existing systems [5,6,7]. First, Moara maintains aggregation trees for different groups adaptively based on the underlying environment and the injected queries to minimize the overall message cost and query response time. Basically, the aggregation tree for a group in Moara is an optimized sub-graph of a global spanning tree, which spans all nodes in the group. By aggregating data over these group-based aggregation trees, Moara achieves lower message cost and response latency for queries compared to other aggregation systems that contact all nodes. Further, we adapt each aggregation tree to deal with dynamism.

Second, Moara's query processor supports composite queries that target multiple groups simultaneously. Composite queries supported by Moara are arbitrary nested set expressions built by using logical operators or and and, (respectively set operations $\cup$ and $\cap$ ) over simple group-predicates. Simple group-predicates are of the form (attribute op value), where $o p \in\{\langle\rangle,, \leq, \geq,=, \neq\}$. Consider our previous example "find top3 loaded hosts where (ServiceX $=$ true) and (Apache $=$ true)", which is a composite query that targets the intersection of two groups - hosts that run service $\mathrm{X}$ and hosts that run Apache. Instead of blindly querying all the groups present in a query, Moara's query processor analyzes composite queries and intelligently decides on contacting a set of groups that minimizes the communication overhead.

We implemented a prototype of Moara by leveraging the FreePastry DHT (Distributed Hash Table) [8] and SDIMS [7] systems. Our evaluation consists of experiments on Emulab [9] and PlanetLab, as well as large-scale simulations. Our experimental results indicate that, compared to previous global hierarchical aggregation systems, Moara reduces response latency by up to a factor of 4 and achieves an order of magnitude bandwidth savings. Our scalability experiments confirm that Moara's overhead for answering a query is independent of the total number of nodes in the system, and only grows linearly with the group size. Finally, we show that Moara can answer complex queries within hundreds of milliseconds in systems with hundreds of nodes under high group churn.

In this work, we focus on efficiently supporting one-shot queries (as opposed to repeated continuous queries) over a common set of groups, since we expect this type of queries to be more common in the kind of infrastructures we are targeting 
at - datacenters and federated computing systems. We expect most users will be performing one-shot queries over common groups (e.g., the same PlanetLab slice, machines in a datacenter, etc) during the time when their service or experiment is running. Further, a user interested in monitoring groups continually can invoke one-shot queries periodically. Our use cases in Section 2 motivate this design decision further.

Any distributed system subjected to dynamism in the environment, suffers from the CAP dilemma [10], which states that it is difficult to provide both strong consistency guarantees and high availability in failure-prone distributed settings. Moara treads this dilemma by preferring to provide high availability and scalability, while providing eventual consistency guarantees on aggregation results. This philosophy is in line with that of existing aggregation systems such as Astrolabe [6] and SDIMS [7]. Moara could also allow the use of metrics proposed by Jain et al. [11,12] in order to track the imprecision of the query results; however, studying these is beyond the scope of the current paper.

\section{Motivation and Use Cases}

We highlight the need for on-demand flexible querying and for dealing with dynamism by presenting two motivating scenarios - data centers and federated infrastructures.

Consolidated Data Centers: In the last few years, medium and large-scale enterprises have moved away from maintaining their own clusters, towards subscribing to services offered by consolidated data centers. Such consolidated data centers consist of multiple locations, with each location containing several thousands of servers [1]. Each server runs heterogeneous operating systems including virtual machine hosts. While such consolidation enables running unified management tasks, it also introduces the need to deal with scale.

Workloads on these data centers typically include Terminal Services, SOA-based transaction workloads (e.g., SAP), and Web 2.0 workloads, e.g., searching and collaboration. Figure 1 presents some on-demand one-shot queries that data center managers and service owners typically desire to run on such a virtualized enterprise. Several of these one-shot queries are for aggregating information from a common group of nodes including cases where groups are expressed as unions of groups (e.g., the third query in table), or intersections (e.g., the last query). We would like to generalize this to provide managers with a powerful tool supporting flexible queries using arbitrarily nested

\begin{tabular}{|c|c|}
\hline Tasks & Queries \\
\hline Resource Allocation & Average utilization for servers belonging to (i) floor F, (ii) cluster C, (iii) rack R \\
\cline { 2 - 2 } & Number of machines/VMs in a given cluster C \\
\hline VM Migration & Average utilization of VMs running application X version 1 or version 2 \\
\cline { 2 - 2 } & List of all VMs running application X and are VMWare based \\
\hline Auditing/Security & Count of all VMs/machines running firewall \\
\cline { 2 - 2 } & Count of all VMs running ESX server and Sygate firewall \\
\hline Dashboard & Max response time for Service X \\
\cline { 2 - 2 } & Count of all machines that are up and running Service X \\
\hline Patch management & List of version numbers being used for service X \\
\cline { 2 - 2 } & Count of all machines that are in cluster C and running service X.version Y \\
\hline
\end{tabular}

Fig. 1. Illustrative Queries for Managing the Virtualized Enterprise 
unions and intersections of groups. In addition, these workloads vary in intensity over time, causing considerable dynamism in the system, e.g., terminal services facing high user turnaround rates.

Federated Computing Infrastructures: In today's federated computing infrastructures such as PlanetLab [2] and global Grids [4], as well as in proposed infrastructures, e.g., GENI [3], users wish to query current statistics for their distributed applications or experiments. For instance, PlanetLab creates virtual subgroups of nodes called "slices" in order to run individual distributed applications. Monitoring is currently supported by tools such as CoMon [13] and Ganglia [14], which periodically collect CPU, memory, and network data per slice on PlanetLab [2]. Due to their periodic nature, they are not open to on-demand queries that require up-to-date answers. Further, increasing the frequency of data collection is untenable due to storage and communication costs.

In contrast to the above systems, we need a system to answer one-shot queries that seek to obtain up-to-date information over a common group of machines, that can be run on-demand or periodically by an end-host, and are flexibly specified. Some examples of our target queries include: number of slices containing at least one machine with CPU utilization $>90 \%$ (basic query), CPU utilization of nodes common to two given slices (intersection query), or free disk space across all slices in a given organization (union query).

Need for Group-based Aggregation: As illustrated by above two target scenarios, we expect that most of the queries are one-shot queries over common groups of machines. Moreover, the predicate in a query specified as a logical expression involves several groups, e.g., some groups in the above examples include the set of nodes in a PlanetLab slice, the set of nodes running a given Grid task, the set of nodes with CPU utilization $>90 \%$, etc. In the worst case, such a group may span the entire system.

In practice though, we expect the group sizes to vary across different queries and with time. In Figure 2(a), we plot the distribution of PlanetLab slice sizes, analyzed from an instance of CoMon [13] data. Notice that there is a considerable spread in the sizes. As many as $50 \%$ of the 400 slices have fewer than 10 assigned nodes, thus a monitoring system that contacts all nodes to answer a query for a slice is very inefficient. If we consider only nodes that were actually in use (where a slice has more than one process
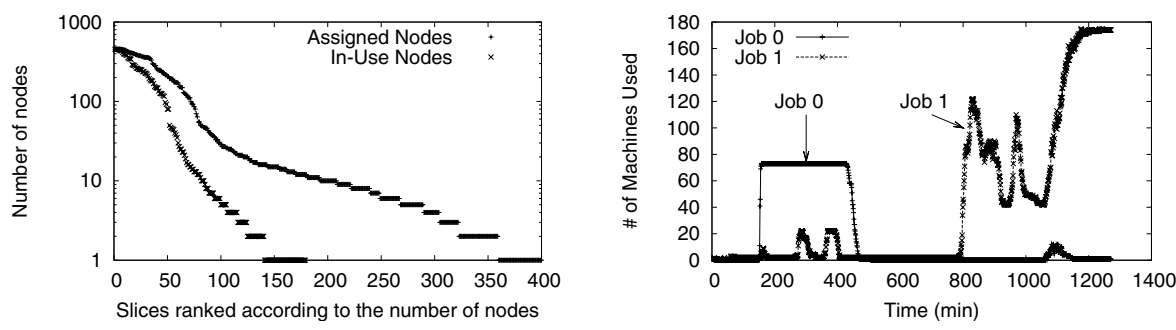

Fig. 2. (a) Usage of PlanetLab nodes by different slices. We show both node assignment to slices and active usage of nodes. Data collected from a CoTop snapshot [15]. (b) Usage of HP's utility computing environment by different animation rendering jobs. We show the number of machines each job uses. 
running on a node), as many as 100 out of 170 slices have fewer than 10 active nodes. In another example case, Figure 2(b) presents the behavior of two jobs over a 20-hour period from a real 6-month trace of a utility computing environment at HP with 500 machines receiving animation rendering batch jobs. This plot shows the dynamism in each group over time.

These trace studies indicate that group sizes can be expected to be varying across time in both consolidated centers as well as in federated computing infrastructures. Thus, an efficient querying system has to avoid treating the entire system as a single group and globally broadcasting queries to all nodes.

\section{The Basics of Moara}

In this section, we first discuss how Moara end-nodes maintain data and how queries are structured. Then we discuss how Moara builds trees for individual groups.

\subsection{Data and Query Model}

Information at each node is represented and stored as (attribute, value) tuples. For example, a machine with CPU capacity of $3 \mathrm{Ghz}$ can have an attribute (CPU-Mhz, 3000). Moara has an agent running at each node that monitors the node and populates (attribute, value) pairs.

A query in Moara comprises of three parts: (query-attribute, aggregation function, group-predicate). The first field specifies the attribute of interest to be aggregated, while the second field specifies the aggregation function to be used on this data. We require this aggregation function to be partially aggregatable. In other words, given two partial aggregates for multiple disjoint sets of nodes, the aggregation function must produce an aggregate that corresponds to the union of these node sets [6,7]. This admits aggregation functions such as enumeration, max, min,sum, count, or top- $k$. Average can be implemented by aggregating both sum and count.

The third field of the query specifies the group of machines on which the above aggregation is performed. If no group is specified, the default is to aggregate values from all nodes in the system. A group-predicate (henceforth called a "predicate") is specified as a boolean expression with and and or operators, over simple predicates of the following form: (group-attribute op value), where op $\in\{\langle\rangle,,=, \leq, \geq, \neq\}$. Note that this set of operators allows us to implicitly support not in a group predicate. Any attribute that a Moara agent populates can be used as either query-attribute or group-attribute.

A simple query contains a simple predicate. For example, the simple predicate (ServiceX $=$ true) defines all machines running ServiceX. Thus, a user wishing to compute the maximum CPU usage across machines where ServiceX is running will issue the following query: (CPU-Usage, MAX, (ServiceX = true)). Alternately, the user could use a composite predicate, e.g., (ServiceX $=$ true and Apache $=$ true). This composite query is defined with set operators $\cup$ and $\cap$.

Note that the query model can be easily extended so that instead of a query-attribute, a querier can specify any arbitrary program that operates upon simple (attribute, value) 
pairs. For example, a querier can specify a program that evaluates (CPU-Available > CPU-Needed-For-App-A) as query-attribute, to see how many nodes are available for the application A. Similarly, group-predicate can be extended to contain multiple attributes by defining new attributes. For example, we can define a new attribute att as (CPU-Available > CPU-Needed-For-App-A), which takes a boolean value of true/false. Then att can be used to specify a group. However, for this paper, we mainly focus on the techniques for efficiently answering the queries for given group-predicates and hence restrict query model to contain only simple attributes.

\subsection{Scalable Aggregation}

We describe here how Moara aggregates data for each group.

DHT trees: For scalability with large number of nodes, groups, and queries, Moara employs a peer-to-peer in-network aggregation approach that leverages the computing and network resources of the distributed infrastructure itself to compute results. These trees are used for spreading queries, and aggregating answers back towards the source node. In our architecture, a lightweight Moara agent runs at each server from which data needs to be aggregated. These agents participate in a structured overlay routing algorithm such as Pastry [8], Tapestry [16], or Chord [17]. These systems allow routing within the overlay, from any node to any other node, based on the IDs of these nodes in the system. Moara uses this mechanism for building aggregation trees called DHT trees, akin to existing systems [7, 18, 19]. A DHT tree contains all the nodes in the system, and is rooted at a node that maps to the ID of the group. For instance, Figure 3 shows the tree for an ID with prefix 000 using Pastry's algorithm with one-bit prefix correction. We choose to leverage a DHT, since it handles physical membership churn (such as failures and join/leave) very modularly and efficiently. Also, we can construct aggregation trees clearly, given a group predicate.

Basics of Resolving Queries: Given a simple query with predicate $p$, Moara uses MD-5 to hash the groupattribute field in $p$ and derives a bit-string that stands for the group ID. The DHT tree for this ID is then used to perform aggregation for this query, e.g., Figure 3 shows the DHT tree for an attribute "ServiceX" that hashes to 000 .

When a simple query is generated at any node in Moara, it is first forwarded to the root node of the corresponding DHT tree via the underlying DHT routing

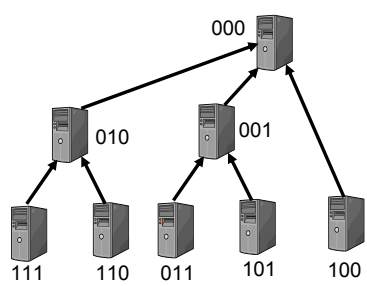

Fig. 3. DHT tree for an ID with prefix 000 mechanism. The root then propagates it downwards along the DHT tree to the leaves. When a leaf receives a query, it evaluates the predicate $p$ in the query (e.g., ServiceX=true). If the result is true, it replies to its parent the local value for the query attribute ( e.g., CPU-Usage). Otherwise, it sends a null reply to its parent. An internal node waits to reply to its parent until all its children have replied or until a timeout occurs (using values in Section 7). Then, it aggregates the values reported by its children, including its own contribution if the predicate is satisfied locally, 
and forwards the aggregate to its parent. Finally, the root node replies to the original querying node with the aggregated value.

Moara Mechanisms: The above "global aggregation" approach has every node in the system receive every query. Hence, it is inefficient in resolving queries targeting specific groups. Moara addresses this via three mechanisms.

First, Moara attempts to prune out branches of the tree that do not contain any node satisfying the predicate $p$. We call this tree a pruned tree or a group tree for $p$. For example, in Figure 3, if nodes 111,110 , and 010 do not satisfy the predicate, then the root does not forward the query to 010. However, this raises a challenge - how do internal nodes know whether any of their descendants satisfy the predicate. For instance, if node 110 decides to install ServiceX and thus satisfies the predicate, the path from the root to this node will need to be added to the tree. Further, if the composition of a group changes rapidly, then the cost for maintaining the group tree can become higher than query resolution costs. Section 4 presents Moara's dynamic adaptation mechanism that addresses this dilemma.

Second, Moara reduces network cost and response latency by short-circuiting the group trees, thus reducing the number of internal tree nodes that do not satisfy the predicate. For instance, in Figure 3, if node 010 does not satisfy the predicate but node 110 does, then the former can be eliminated from the tree by having 110 receive queries directly from the root. Section 5 describes how this reduces the bandwidth cost of aggregating a group with $m$ nodes in a system of $N$ nodes, from $O(m \log N)$ to $O(m)$.

Third, Moara efficiently resolves composite queries involving multiple groups by rewriting the predicate into a more manageable form, and then selecting a minimal set of groups to resolve the query. For example, an intersection query (CPU-Util, avg, (floor=F1 and cluster $=\mathrm{C} 12$ )) is best resolved by sending the query to only one of the two groups - either (floor=F1) or (cluster=C12) - whichever is cheaper. This design decision of Moara is detailed in Section 6 .

\section{Dynamic Maintenance}

Given a tree for a specific group, Moara reduces bandwidth cost by adaptively pruning out parts of the tree, while still guaranteeing correctness via eventual completeness. Eventual completeness is defined as follows - when the set of predicate-satisfying nodes as well as the underlying DHT overlay do not change for a sufficiently long time after a query injection, a query to the group will eventually return answers from all such nodes. For now, we assume that the dynamism in the system is only due to changes in the composition of the groups ("group churn"); we will describe how our system handles node and network reconfigurations (churn in system) later in Section 7.

To resolve queries efficiently, Moara could prune out the branches of the corresponding DHT tree that do not contain any nodes belonging to the group. However, to maintain completeness of the query resolution, Moara can perform such aggressive pruning only if it maintains up-to-date information at each node about the status of branches at that node. For groups with high churn in membership relative to the number of queries (e.g., CPU-Util < 50), maintaining group status at each node for all its branches can consume high bandwidth - broadcasting queries system-wide may be cheaper. For 
relatively stable groups however (e.g., (sliceX $=$ true) on PlanetLab), proactively maintaining the group trees can reduce bandwidth and response times. Instead of implementing either of these two extreme solution points, Moara uses a distributed adaptation mechanism that, at each node, tracks the queries in the system and group churn events from children for a group predicate and decides whether or not to spend any bandwidth to inform its parent about its status.

Basic Pruning Mechanism: Each Moara node maintains a binary local state variable prune for each group predicate. If prune for a predicate is true (PRUNE state), then the branch rooted at this node can be pruned from the DHT tree while querying for that predicate. Whenever a node goes from PRUNE to NO-PRUNE state, it sends a NO-PRUNE message to its parent; the reverse transition causes a PRUNE message to be sent. When the root or an internal node receives a query for this predicate, it will forward the query to only those of its children that are in NO-PRUNE state.

Note that it is incorrect for an internal node to set its state for a predicate to PRUNE based merely on whether it satisfies the predicate or not. One or more its descendants may satisfy the predicate, and hence the branch rooted at the node should continue to receive any queries for this predicate. Further, an internal or a leaf node should also consider the churn in the predicate satisfiability before setting the prune variable. For example, suppose the predicate is (CPU-Util < 50) and a leaf node's utilization is fluctuating around $50 \%$ at a high rate. In this case, the leaf node will be setting and unsetting prune variable, leading to a large number of PRUNE/NO-PRUNE messages.

Due to the above reasons, we define the prune variable as a variable depending on two additional local state variables_-sat and update. sat is a binary variable to track if the subtree rooted at this node should continue receiving queries for the predicate. Thus sat is set to 1 (SAT) if either the local node satisfies the predicate or any child node is in NO-PRUNE state.

update is a binary state variable that denotes whether the node will update its prune variable or not. So, when update $=1$ (UPDATE state), the node will update the prune variable; but, when update $=0$ (NO-UPDATE state), the node will cease to perform any updates to the prune variable irrespective of any changes in the local satisfiability, or any messages from its children. In other words, a node does not send any PRUNE or NO-PRUNE messages to its parent when it is in NO-UPDATE state. So, to ensure correct operation, a node can move into NO-UPDATE state only after setting prune = 0 . This guarantees that its parent will always send the queries for the predicate to this node. Formally, we maintain the following invariants:

$$
\begin{array}{r}
\text { update }=1 \text { AND } \text { sat }=1 \Longrightarrow \text { prune }=0 \\
\text { update }=1 \text { AND } \text { sat }=0 \Longrightarrow \text { prune }=1 \\
\text { update }=0 \Longrightarrow \text { prune }=0
\end{array}
$$

The transition rules for the state machine at each node is illustrated in Figure 4 Note that a node sends a status update message to its parent whenever it moves from PRUNE to NO-PRUNE state or vice-versa. This state machine ensures the following invarianteach node in the system performs at least one of the following: (a) sends status updates upwards to its parent, or (b) receives all queries from its parent. This invariant suffices to guarantee eventual completeness because after the group stops changing, any node 
that satisfies the predicate will be in SAT state. Therefore, the node and its ancestors will all be in NO-PRUNE state, and thus the node will receive the next query. Our technical report [20] elaborates with pseudo-code how Moara evaluates each variable.

Adaptation Policy: To decide the transition rules for the update state variable, Moara employs an adaptation mechanism that allows different policies. Our goal is to use a policy that minimizes the overall message cost, i.e., sum of both update and query costs. In Moara, each node tracks the total number of recent queries and local changes it has seen (in the tree) -

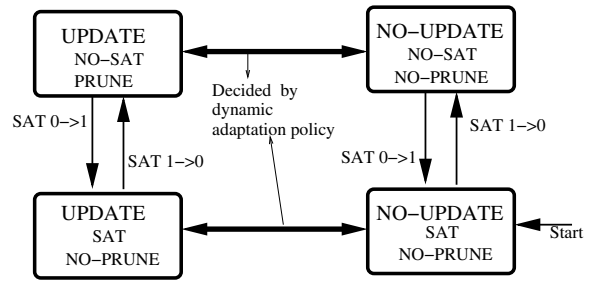

Fig. 4. State machine for dynamic adaptation mechanism we discuss details of how to keep track of recent queries and local changes in our technical report [20]. Each node keeps two query counts - $q_{n}$, the number of queries recently received by the system while the node is in NO-SAT state, and $q_{s}$, the number of recent queries received by the system while it was in SAT state. The node also keeps track of the number of times the sat variable toggled between 0 and 1 , denoted as $c$.

A node in NO-UPDATE state would exchange a total of $B_{N U}=2 \times\left(q_{n}+q_{s}\right)$ messages with its parent (two per query), while a node in UPDATE state would exchange $B_{U P}=c+2 \times q_{s}$ messages (one per change, and two per query). Thus, to minimize bandwidth, the transition rules are as follows: (1) a node in UPDATE state moves to NO-UPDATE if $B_{N U}<B_{U P}$, i.e., $2 \times q_{n}<c$; (2) a node in NO-UPDATE state moves to UPDATE if $B_{N U}>B_{U P}$, i.e., $2 \times q_{n}>c$. In order to avoid flip-flopping around the threshold, we could add in hysteresis, but our current design performs well without it.

One corner issue with the above approach is that when a node is in the PRUNE state, it does not receive any more queries and thus cannot accurately track $q_{n}$. Note that this does not affect the correctness (i.e., eventual completeness) of our protocol but may cause unnecessary status update messages. To address this, the root node of an aggregation tree in Moara assigns a sequence number for each query and sends that number piggybacked along with the queries. Thus, any node that receives a query with sequence number $s$ is able to track $q_{n}$ using the difference between $s$ and its own lastseen query sequence number.

State Maintenance: By default, each node does not maintain any state, which is considered as being in NO-UPDATE state. A node starts maintaining states only when a query arrives at the node. Without dynamic maintenance, merely maintaining pruned trees for a large number of predicates (e.g., a tree for each slice in the PlanetLab case or a tree for each job in the data center) could consume very high bandwidth in an aggregation system. With dynamic maintenance, pruning is proactively performed for only those predicates that are of interest at that time. Once queries stop, nodes in the aggregation tree start moving into NO-UPDATE state with any new updates from their children and hence stop sending any further updates to their parents. 
We note that a node in NO-UPDATE state for a predicate can safely garbage-collect state information (e.g., predicate itself, recent events information, etc) for that predicate without causing any incorrectness in the query resolution. So, once a predicate goes out of interest, eventually no state is maintained at any node and no messages are exchanged between nodes for that predicate. Several policies for deciding when to garbage-collect state information are possible: we could 1) garbage-collect each predicate after a timeout expires, 2) keep only the last $k$ predicates queried, 3) garbage-collect the least frequently queried predicate every time a new query arrives, etc. However, studying these policies is beyond the scope of this paper. We also note that we do not consider DHT maintenance overhead. In addition, note that global aggregation trees are implicit from the DHT routing and hence require no separate maintenance overhead.

Finally, since Moara maintains state information for each predicate, it could be more efficient if we aggregated different predicates. For example, predicates such as CPUUtil $>50$, CPU-Util $>60$, and CPU-Util $>70$ could be aggregated as one predicate, CPU-Util $>50$, so that Moara could maintain only one tree. This design choice requires careful study on the tradeoff between the state maintenance overhead and the bandwidth overhead incurred by combining different trees with the same attribute. This is outside of the scope of this paper, since we focus on the tradeoff of the bandwidth overhead based on the query rate and the group churn rate.

\section{Separate Query Plane}

Given a tree that contains $m$ predicate-satisfying nodes, using the pruned DHT trees of the previous section may lead to $O(m \log N)$ additional nodes being involved in the tree. These extra nodes would typically be internal tree nodes that are forwarding queries down or responses up the tree, but which do not satisfy the predicate themselves. This section proposes modifications to the protocol described in Section 4 in order to reduce the traffic through these internal nodes.

Our idea is to bypass the internal nodes, thus creating a separate query plane which involves mostly nodes satisfying the predicate. This optimizes the tree that we built (Section 4) further by eliminating unnecessary internal nodes. This reduces the tree to contain only $O(m)$ nodes, and thus resolves queries with message costs independent of the number of nodes in the system. Note that this technique has similarities to adaptations of multicast trees (e.g., Scribe [18]), but Moara needs to address the challenging interplay between dynamic adaptation and this short-circuiting.

To realize a separate query plane, each node uses the states, constraints and transitions as described in Section 4 . In addition, each node runs operations using two locally maintained sets: (i) updateSet is a list of nodes that it forwards to its parent; (ii) qSet is a list of children or descendant nodes, to which it forwards any received queries. We consider first, for ease of exposition, modified operations only for nodes in the UPDATE state. When a leaf node in UPDATE state begins to satisfy the tree predicate, it changes to SAT state as described in Section 4 and sets its UpdateSet to contain its ID. In addition, when sending a NO-PRUNE message to its parent, it also sends the updateSet. Each internal node in turn maintains its $q$ Set as the union of the latest received updateSets from all its children, adding its own ID (IP and port) if the tree predicate is 
satisfied locally. The leaf nodes do not need to maintain $q$ Sets since they do not forward queries.

Finally, each internal node maintains its updateSet by continually monitoring if $|q S e t|$ $<$ threshold, where threshold is a system parameter. If so, then updateSet is the same as $q$ Set, otherwise updateSet contains a single element that is the node's own ID regardless of whether the predicate is satisfied locally or not. Whenever the updateSet changes at a node and is non-empty, it sends a NO-PRUNE message to its parent along with the new updateSet informing the change. Otherwise, it sends a PRUNE message.

The above operations are described assuming that all nodes are in UPDATE state. When a node is NO-UPDATE state, it maintains $q$ Set and updateSet as described above, but does not send any updates to its parent. For correctness, a node moving from UPDATE to NO-UPDATE state sends its own ID along with the NO-PRUNE message to its parent so that it receives future queries.

If parameter threshold $=1$, the above mechanisms produce the pruned DHT tree described in Section 4, while threshold $>1$ gives trees based on a separate query plane. This is because with threshold $=1$, an internal node that receives an updateSet from any of its children will pass along to its parent an updateSet containing its own ID, even if the predicate is not satisfied locally. However, with threshold $>1$, the only internal nodes that do not satisfy the predicate locally but receive queries, are ones that are maintaining a $q$ Set of size $\geq$ threshold. Such nodes are required to receive queries so that they

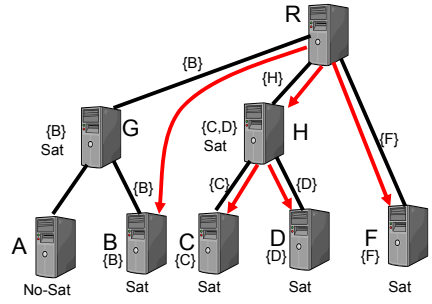

Fig. 5. Separate Query Plane for threshold $=1$. We assume all nodes are in UPDATE mode. Each node's qSet is shown next to it, and updateSet on the link to its parent. can be forwarded to its descendants. However, the tree bypasses several other nodes that do not satisfy the predicate, thus obtaining bandwidth savings. Specifically, an internal node that has $\mid$ qSet $\mid<$ threshold and does not satisfy the predicate, does not include its own ID in the updateSet, and thus does not receive queries.

Having a high value of threshold in the system bypasses several internal nodes in the tree. However, this comes at the expense of a higher update traffic since any updateSet changes need to be communicated to the parent. Figure 5 shows an example with threshold $=1$. The overhead of forwarding a query in the separate query plane is $O(m)$ for a group with $m$ nodes, independent of system size (details can be found in our technical report [20]).

Our SQP design with updateSet and qSet variables at nodes, as described above, allow us to easily use the adaptation policy rules described in Section 4 . Further details on this can be found in our technical report [20].

\section{Composite Queries}

So far, we have described how to build and maintain a single tree corresponding to one simple predicate. We now describe how a query with a composite predicate is satisfied. 
Specifically, we first expand on the multiple possible trees, one tree per simple predicate in the composite query, that such a query entails (Section 6.1). Then, we explain how Moara plans a given query (Section 6.2), and how it selects a low-cost groups of nodes to execute a given composite query (Section 6.3).

\subsection{Maintaining Multiple Trees}

Section 4 explains the maintenance of trees for simple predicates, starting from the time a predicate is first encountered. If this predicate does not reappear again in subsequent queries in the system, then all nodes in the tree will eventually move to NO-UPDATE state (due to group churn events), and thus there will be no load, either query or update, along the tree. Thus, Moara trees become silent and incurs zero bandwidth cost if not used, obviating the need to explicitly delete trees for simple predicates. Furthermore, Moara does not maintain trees for composite queries, since these might be exponentially large in number - instead, it decides which simple predicate trees (existing or not) will be selected to execute a given composite query. This decision process is described next.

\subsection{Composite Query Planning}

Consider the following composite query: "find the average free memory across machines where service X and Apache are running". Suppose we have one group tree for (ServiceX=true) and another tree for (Apache=true). A naïve way to resolve the query would be to query both trees in parallel. However, we observe that bandwidth can be saved, without compromising completeness of answers, by (1) sending the query to any one of the trees (because it is an intersection query), and (2) choosing the tree that incurs a lower query cost.

Based on this observation, Moara answers arbitrary nested queries involving and and or boolean expressions across simple predicates by selecting a small cover. A cover for a given composite query $Q$ is defined as a set of groups (selected from among simple predicates inside $Q$ ) which together contain all nodes that satisfy the composite predicate in $Q$. Thus, we only need to send $Q$ to a cover to obtain a complete answer.

We can compute a cover for a query $\mathrm{Q}$ by exploring the boolean expression structure recursively as follows:

- $\operatorname{cover}(\mathrm{Q}=$ "A" $)=\{\mathrm{A}\}$ if $\mathrm{A}$ is a predefined group.

- $\operatorname{cover}(\mathrm{Q}=$ "A or $\mathrm{B}$ ” $)=\operatorname{cover}(\mathrm{A}) \cup \operatorname{cover}(\mathrm{B})$.

- $\operatorname{cover}(\mathrm{Q}=“ \mathrm{~A}$ and $\mathrm{B} ")=\operatorname{cover}(\mathrm{A}), \operatorname{cover}(\mathrm{B}), \operatorname{or}(\operatorname{cover}(\mathrm{A}) \cup \operatorname{cover}(\mathrm{B}))$.

For example, for a query with expression ( $(\mathrm{A}$ and $\mathrm{B})$ or $\mathrm{C})$, the above rules derive $\{\mathrm{A}, \mathrm{C}\},\{\mathrm{B}, \mathrm{C}\}$, and $\{\mathrm{A}, \mathrm{B}, \mathrm{C}\}$ as possible covers. We call such covers as structural covers since we infer them from the structure of the boolean expression.

Once the query originating node calculates the cover for a given query $Q$, the composite query is forwarded to the roots of trees corresponding to each group in the cover, the answers from these trees are aggregated, and finally returned to the querying node. Notice that it is possible for some node(s) to receive multiple copies of the query, if they are present in multiple trees which appear in the cover for $Q$. Such nodes reply with the 
attribute value to only one of the trees they are present in, eliminating duplicate answers. This requires nodes to remember the query ids (based on sender IP and sequence number). Such information is cached for 5 minutes in our Moara implementation.

To further save on bandwidth, we would like to select a low-cost cover. This is done by minimizing both the number of groups in the selected cover, as well as the total cost of querying this cover. We explore below three ways of deriving a low-cost cover: (1) structural optimizations, which rewrite the nested query to select a low-cost structural cover consisting of simple predicates that already appear within the query, (2) estimates of query costs for individual trees, and (3) semantic optimizations, which take into account semantic information obtained from users or query attributes.

\subsection{Query Optimization: Finding Low-Cost Covers}

Given a composite query, Moara first transforms it into a Conjunctive Normal Form (CNF) expression using distributive laws of and and or operators. A CNF form is a two level expression of and's across a series of or terms.

It is important to notice that in the CNF form of a composite predicate for query $Q$, each series of or terms is a possible cover - this is due to the same reason as our intersection optimization explained earlier. Thus, if Moara can evaluate the query cost of each of these structural covers (as a sum of the query costs for all sets in the cover), then it can select the minimal cost cover for executing the query $Q$. We will describe query cost calculation soon, but before that we give an example of the query rewriting. The proof of why the CNF form gives the minimal-cost cover for a composite predicate can be found in our technical report [20].

Figure 6 shows an example transformation. Consider a query targeting $((\mathrm{A}$ or $\mathrm{B})$ and $(\mathrm{A}$ or $\mathrm{C})$ ) or $\mathrm{D}$. Moara first transforms the expression to the equivalent CNF: (A or B or D) and (A or C or D). Moara chooses one cover between the two structural covers either $\{A, B, D\}$ or $\{A, C, D\}$, whichever has a lower cost.

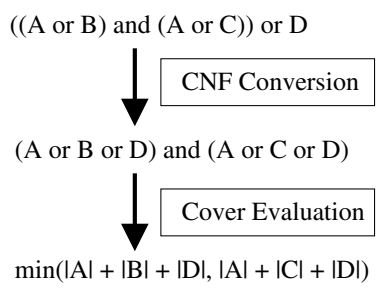

Fig. 6. Example query processing

Estimating Query Costs for Trees: In order to enable low-cost cover calculation, the root node of each tree for a simple predicate continually maintains the query cost for that tree. The query cost is fetched by the querying node and used in the low-cost cover calculation described above. Within the tree, the cost for each query is simply $2 \times n p$, where $n p$ is the number of nodes in NO-PRUNE state. The values of $n p$ are aggregated continually up the tree. Each internal node stores this count for its own subtree, modifies the count according to its own state, and piggybacks this information atop all updates and query responses to its parents. Although this lazy updating of the counts means the query costs may be stale at times, this only affects communication overhead, but not the correctness of the response.

Using Semantic Optimizations: If semantic information is available about the groups, then Moara can further optimize the communication costs by choosing a better cover. We explore two kinds of semantic information in our system: (i) information from 
description of the group, and (ii) user supplied semantic information. For example, consider two groups A and B defined as follows: $A=\{$ nodes with memory $<2 G$ \} and $B=$ nodes with memory $<1 G\}$. Then, we can infer from these definitions that $B \subseteq A$. In our technical report [20], we detail a variety of semantic relations between two groups Moara exploits and how Moara optimizes further to obtain a low-cost cover.

\section{Implementation and Evaluation}

We have built a prototype of Moara using SDIMS [7] and FreePastry [8]. All other Moara protocols, described in Section 3 through Section 6, are built atop these systems. Here, we discuss our implementation details and evaluation methodology.

Moara Front-End: The Moara front-end is a client-side interface of Moara. It includes an interactive shell, a query parser, and a query optimizer. Through the interactive shell, a user can submit SQL-like aggregation queries to Moara. The query parser parses the queries, and the query optimizer determines the groups that need to be queried through the algorithm described in Section 6. Once the front-end determines the groups to be queried, it generates a sub-query for each group. Each sub-query is resolved exactly the same way as a normal query, except that the front-end waits until it receives all the results from sub-queries, aggregates the results returned by the sub-queries, and returns the final aggregate to the user.

Reconfigurations: To handle reconfigurations, we leverage the underlying FreePastry mechanism for failure detection and neighbor set repair. Further details can be found in our technical report [20].

Evaluation Environments: We use simulation, Emulab, and PlanetLab, and choose a suitable environment to evaluate each of our design choices. We use simulation exclusively for measuring bandwidth consumption in a large-scale environment. We use Emulab and PlanetLab to mainly measure the latency in realistic environments, namely, a medium-scale datacenter (Emulab) and a wide-area infrastructure (PlanetLab).

For each design choice (group-based aggregation, dynamic maintenance, separate query plane, and composite query processor), we choose the evaluation environments that are most suitable. First, we evaluate group-based aggregation on Emulab and PlanetLab, since group-based aggregation is designed to reduce both latency and bandwidth consumption. Second, we evaluate dynamic maintenance and separate query plane using simulation, since both mechanisms are designed for bandwidth optimization and have wide choices of parameters. However, we evaluate the separate query plane on Emulab as well to measure the latency. Lastly, we evaluate our composite query processor on Emulab, since it only affects latency.

Workload: The workload is characterized by two factors - group churn rate and query rate. First, since a group is defined over a particular attribute, the group churn rate depends on how dynamic the attribute is (e.g., a group of ( $\mathrm{OS}=$ Linux) is likely to be static, while a group of (CPU-util $<60 \%$ ) is likely to be dynamic). Second, the query rate depends on the usage of Moara and is expected to vary widely. For example, a datacenter operator might typically query a group once an hour on a day, but several times a 
minute on days with high workloads or unscheduled downtimes. Thus, we parameterize these factors and present the performance of Moara over the parameter range.

\subsection{Simulation Results}

We perform simulation experiments to measure the bandwith overhead of Moara's dynamic tree maintenance and separate query plane. Our simulations are performed with the FreePastry simulator environment, simulating up to 16,384 nodes. Each node maintains an attribute $A$ with value $\in\{0,1\}$. All queries are simple queries for ( $A$, SUM, $A=1$ ), which counts the number of nodes where $A$ is set to 1 .

Dynamic Maintenance: To study the dynamic maintenance mechanism under different workload types, we stress the system by injecting two types of events - query events and group churn events - at different ratios. For example, a query:churn ratio of 0:500 represents an extreme type of workload where there is high group churn, but no queries at all. On the other hand, the query:churn ratio of 500:0 represents the other extreme where there is high query rate, but no group churn. Each group churn event selects $m$ nodes at random, and toggles the value of their attribute $A$. The value of $m$ determines the "burst size" of attribute churn. We fix the total number of events to 500, and randomly inject query or group churn events at the chosen ratio. All data points are averaged over 3 runs.

Figure 7 shows the average number of messages per node in Moara under various query:churn ratios, in a system of 10,000 nodes with $m=2000$ sized group churn events. In addition to Moara, we also plot the number of messages generated by two other static approaches that lie at the opposing extremes. These are: 1) the Global approach, where no group trees are maintained and queries are sent to all the nodes on the DHT trees, and 2) Moara

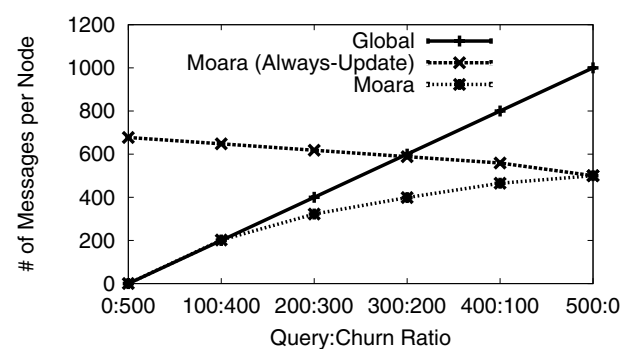

Fig. 7. Bandwidth usage with various query-tochurn ratios (Always-Update) approach, where a tree is aggressively maintained by having each child send an update to its parent on each attribute churn event.

The Global approach is inexpensive when there are fewer queries in the system, since it avoids the overhead of tree maintenance. On the other hand, with a high-query:lowchurn ratio, Moara (Always-Update) performs well because it always maintains group trees and hence incurs lower traffic than Global approach. The plots show that Moara meets or lowers the message overhead in comparison to either of these extreme design choices, at all values of query:churn ratios. When group churn is high, Moara suppresses attribute churn events from propagating to other nodes. With more queries than group churn events, Moara reduces query cost by maintaining trees aggressively. Thus, Moara is able to adapt to various workload patterns.

Separate Query Plane: In Figure 8(a), we plot the query cost against the number of nodes in the system for different threshold values and different group sizes. Note that 

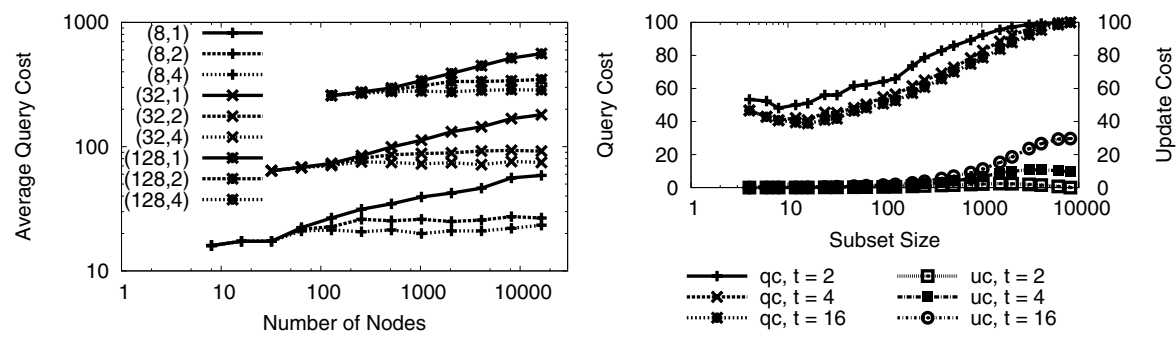

Fig. 8. (a) Bandwidth usage with (threshold $>1$ ) and without the separate query plane (threshold=1) for different group sizes. Each line represents a (group size, threshold) pair (b) Query costs (qc) and update costs (uc) of the separate query plane in a 8192-node system.

the threshold value of 1 implies the absence of a separate query plane, while higher threshold values create a separate query plane (refer to Section 5). For this experiment, we do not introduce any group churn during the experiment. We perform 1,000 queries and compute the average of the query cost. Even though there is no group churn, there are updates sent by nodes to their parents as they move into UPDATE state with the first query message. We count those messages as the update cost.

Figure $8(\mathrm{a})$ shows that without the separate query plane (threshold $=1$ ), the query cost increases logarithmically as the total system size is raised. However, while maintaining a separate query plane (threshold $>1$ ), the query cost reaches a constant value and stays flat, independent of the number of nodes in the system. While increasing the value of threshold decreases query cost, it can lead to more update messages as discussed in Section 5 In Figure 8(b), we plot the query costs for different threshold values as a percentage of the query cost for threshold $=1$ and also plot the percentage increase in the update costs in comparison to threshold $=1$. From these two plots, we observe that (1) with small groups and large total nodes (e.g., 8192 total nodes with group size $=8$ or 32), using a query plane saves more than $50 \%$ bandwidth in query costs, and (2) while using a higher value of threshold does reduce bandwidth, the savings are marginal beyond a threshold of 2 and can incur higher update costs at large group sizes.

\subsection{Emulab Experiments}

In this section, we study both the latency and communication overhead of Moara under a real deployment scenario in Emulab, that emulates a medium-scale datacenter. Specifically, we evaluate three different workloads. First, we study performance of Moara when querying groups of static attributes (e.g., OS = Linux). We vary the size of groups and show the benefits of using Moara. Second, we study Moara with groups defined over dynamic attributes (e.g., CPU-util $<60 \%$ ). We stress Moara by varying the frequency of changes. Third, we study composite queries with varying numbers of groups per query.

Methodology: We create a network of 50 machines on a $100 \mathrm{Mbps}$ LAN and instantiate 10 instances of Moara on each machine, thus emulating a 500 node Moara system. Each experimental run is started with one bootstrap node, followed by a batch of 100 

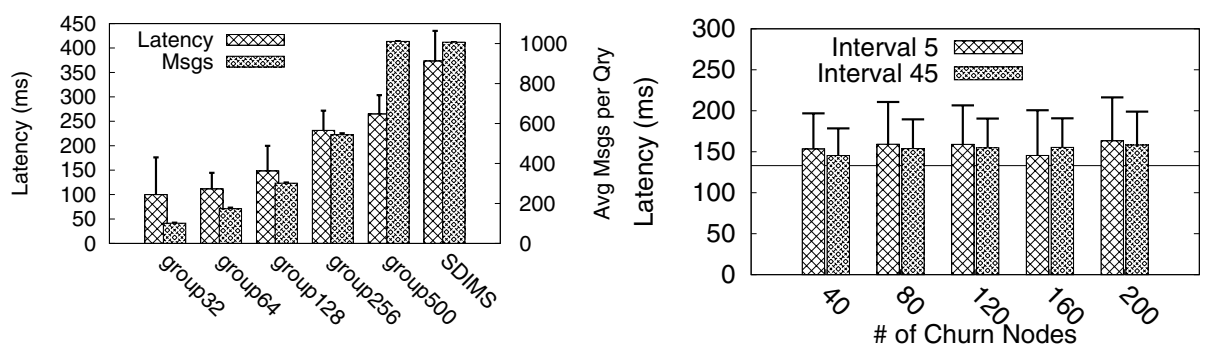

Fig. 9. (a) Latency and bandwidth usage with static groups (b) Average latency of dynamically changing groups. The horizontal line shows the average latency with a static group of the same size.

new instances joining after intervals of 10 seconds each. After the last join, we wait an additional 5 minutes to warm up before initiating queries and group churn from a Moara node. Since we are mainly interested in per-query latency and bandwidth consumption, we fix the query rate and repeat the same query multiple times. As previously, each node maintains one binary attribute $A$. Our default query is a count, providing the number of nodes with $A=1$. All data points are the average of 3 runs.

Static Groups: Figure 9(a) compares the performance of Moara (with separate query plane) w.r.t. both latency and bandwidth. We vary the group sizes and query 100 times for each experiment. In addition, we compare this performance against an approach where a single global tree is used system-wide - this is labelled as the SDIMS approach in the plot. As we can see from the figure, Moara's latency and bandwidth scale with the size of the group. The savings are the most significant for small groups (e.g., set32 which has 32 nodes), where the savings compared to the SDIMS approach are up to $4 \mathrm{X}$ in latency and 10X in bandwidth. The latency is reduced due to the use of separate query plane because of short-circuiting long chains of intermediate nodes.

Dynamic Groups: We study the effect of group churn due to attribute-value changes at individual nodes. We considered a group of 100 nodes, with group churn controlled by two parameters churn and interval. Every interval seconds, we randomly select churn nodes in the group to leave, and churn nodes outside the group to join.

Figure 9(b) shows the effect on query latency, of different churn values (x-axis) for two different interval values. Queries are inserted at the rate of one query per second, and the data points are averages of 100 queries per run. The plot shows that Moara's query latency is not affected significantly by group churn - (1) even when we increase the group churn rate by a 9-fold factor from Interval=45 to interval=5, Moara experiences only a small increase in latency, and (2) the latency stays low, and around $150 \mathrm{~ms}$ even when the entire group membership changes every 5 seconds.

Figure 10(a) provides an insight into the workings of Moara under the above workload, for interval $=5$, churn $=160$. Notice that the spikes in query latency occur once every 5 seconds, around the time that the group churn batch occurs. However, notice that (1) the peak latency stays within $300 \mathrm{~ms}$, and (2) Moara query latency stabilizes 

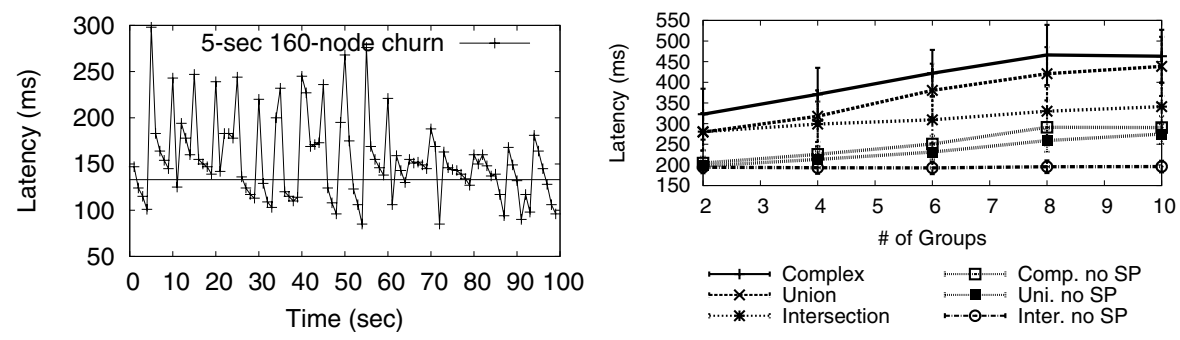

Fig. 10. (a) Latency over time with a dynamically changing group. The horizontal line shows the average latency with a static group of the same size. (b) Latency with composite queries.

very quickly after each group churn batch, typically within 1-2 seconds. Thus Moara shows high resiliency to dynamism due to rapidly occurring attribute-value changes.

Composite Queries: The experiments so far have focused on single groups in Moara. Here, we microbenchmark the performance of Moara on composite queries. Assuming $S_{1}, S_{2}, \ldots, S_{n}$ are simple single predicate groups, we study three types of composite queries: (1) Intersection queries of the form $S_{1} \cap S_{2} \cap \ldots \cap S_{n}$, for different values of $n$; (2) Union queries of the form $S_{1} \cup S_{2} \cup \ldots \cup S_{n}$, for different values of $n$; and (3) Complex queries, which are structured as $T_{1} \cap T_{2} \cap \ldots \cap T_{m}$, where each $T_{i}$ is a union of multiple groups. These experiments suffice to characterize Moara's performance since the query optimization reduces all query expressions to one of the three. Each basic group $S_{i}$ consists of 50 nodes selected at random. The complex expression we use 1 is $T_{1} \cap T_{2} \cap T_{3}$, and each $T_{i}$ is a union of $n$ basic groups for different values of $n$. Figure 10(b) plots the latency for above three types of queries with different values of $n$. For composite queries, recall that Moara first sends size probes to root nodes of group trees, in order to make a query optimization decision. Thus, we plot not only the total latency of a Moara query, but also the latency excluding the time to finish the size probes. Each data point is averaged over 300 queries.

First, notice that the average completion times of all queries, including queries with up to 10 groups, is less than $500 \mathrm{~ms}$. For intersection queries, the completion times excluding time for size probes (plot line "Inter. no SP") do not depend on the size of the expression. This is because Moara selects only one of these groups to propagate the query. Although size probes are sent in parallel, the latency for size probes increases slightly since Moara waits until the slowest probe response arrives. For union queries, the total completion time of a query rises gradually with the size of the expression, as Moara needs to contact all groups (two "Union" plots). Finally, the completion time for complex queries is only slightly more than that of union queries, since Moara's query optimization selects only one of $T_{i}$ 's. The additional latency is caused by two factors: (a) the time taken for size probes is higher as we have to query the sizes for larger number of groups, and (b) a complex set expression adds more overhead at each node, because each node evaluates the entire complex expression.

\footnotetext{
${ }^{1}$ We found that the number of $T_{i}$ 's has little effect on latency because Moara queries only one of all $T_{i}$ 's.
} 


\subsection{PlanetLab Experiments}

Methodology: We deploy Moara atop 200 PlanetLab nodes, which span several continents. Each PlanetLab node runs one instance of Moara. The instances are started sequentially, the system is given 5 minutes to warm up, and then a series of queries is injected from a Moara front-end running on a local machine. In order to study the behavior of Moara's query latency in-depth, we perform experiments on only one group at a time, but for different sizes of this group. Each experiment involves a total of 500 queries injected 5 seconds apart. All plotted data points are the average of 3 runs. We do not timeout on queries, in order to obtain complete answers.

Query Response Latency: Figure 11 plots the cumulative fraction of replies received as a function of time since query injection. The plot shows the responsiveness of Moara in a wide-area setting - even with as many as 100 nodes in the group, the median answer is received back within 1-2 seconds, while $90 \%$ of the answers are received within 5 seconds. Our technical report presents more results with different groups [20].

\section{Moara versus Centralized Aggre-}

gation: Figure 11 compares Moara against a centralized approach which maintains no trees but has the Moara front-end directly query all nodes in parallel regardless of whether they satisfy the given predicate or not (labelled "Central"). The response for a query from this centralized aggregator is considered complete when the cen-

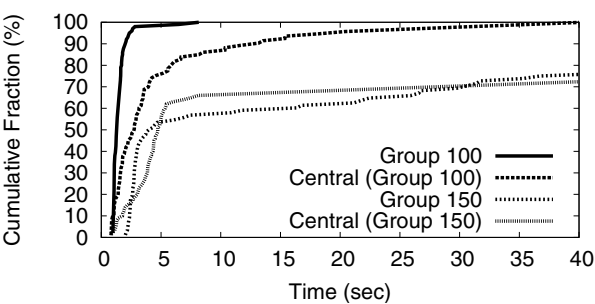

Fig. 11. Moara vs. Centralized Aggregator tralized aggregator has received a response from every node regarding the query. The figure plots the cumulative fraction of replies received as a function of time since query injection. This plot illustrates that the comparison between the centralized aggregator and Moara is akin to the comparison of "the tortoise and the hare". For both groups of size 100 and 150 , we notice that the centralized aggregator obtains initial replies faster than Moara, but then it slows down waiting for the remainder of the query answers from nodes.

Our analysis reveals that the latency of the centralized aggregator is affected by the slowest node or link in the whole system, while the latency of Moara is only affected by the slowest node or link in the group. Thus, Moara is faster overall in obtaining a large fraction of replies. Our technical report further discusses this result [20].

\section{Related Work}

PlanetLab has several management tools in use, such as CoTop, CoMon, etc [15]. However, none of the tools addresses scalability and expressive queries simultaneously. Several distributed systems have been proposed for aggregating data. Astrolabe [6] provides a generic aggregation abstraction, but uses a single static tree and hence has limited scalability with the number of metrics. SDIMS [7] constructs multiple trees for scalability with the number of metrics, but assumes a single group of the entire system. PIER [21] 
supports recursive SQL-style queries, but does not leverage in-network aggregation. Huebsch et al. [22] present a way to optimize global aggregation queries, while Moara optimizes multiple group-based aggregation trees. Seaweed [5] focuses on dealing with data unavailability. MON [23] supports one-shot queries and constructs query trees ondemand, but does not support expressive queries. Finally, Ganglia [14] uses a single hierarchical tree, but collects all data without in-network aggregation.

Structured overlay based multicast systems such as Scribe [18], SAAR [24], and SelectCast [25] bear some similarities with Moara, e.g., path collapsing of Scribe [18], the shared control plane idea of SAAR [24], and predicate-based multicast of SelectCast. However, all these system focus on building efficient trees for multicast where maintenance overhead is assumed to be much smaller than the data plane costs. CUP [26] and Shruti [27], while proposing adaptation techniques to reduce query cost, addresses a different optimization problem than us. In these systems, queries are only spread down to the nodes where updates are also propagated to (rendezvous points). Moara uses updates for pruning the group trees and queries are sent to all predicate-satisfying nodes.

\section{Conclusion}

In this paper, we have presented the design and evaluation of Moara, a group-based aggregation system. Moara achieves scalability with increasing numbers of machines, injected queries, and groups, by: (1) intelligently resolving composite query expressions, (2) constructing single-attribute aggregation trees that perform in-network aggregation, and (3) dynamically maintaining group trees based on query rates and group churn rates, thus reducing bandwidth consumption. Our experimental evaluations using simulations and deployments atop Emulab and PlanetLab demonstrate the effectiveness of Moara in answering queries accurately within hundreds of milliseconds across hundreds of nodes, and with low per-node bandwidth consumption.

\section{References}

1. HP: HP Data Centre Consolidation, http://h20331.www2 .hp.com/enterprise/ cache/141741-0-0-225-121.html

2. PlanetLab, http://www.planet-lab.org/

3. NSF: The NSF GENI Initiative, http://www.nsf.gov/cise/geni/

4. Foster, I.T.: The Grid 2003 Production Grid: Principles and Practice. In: Proc. HPDC-13 (2004)

5. Narayanan, D., Donnelly, A., Mortier, R., Rowstron, A.: Delay Aware Querying with Seaweed. In: Proc. VLDB (2006)

6. Renesse, R.V., Birman, K.P., Vogels, W.: Astrolabe: A Robust and Scalable Technology for Distributed System Monitoring, Management, and Data Mining. ACM Trans. on Comp. Syst. 21(2), 164-206 (2003)

7. Yalagandula, P., Dahlin, M.: A Scalable Distributed Information Management System. In: Proc. SIGCOMM (2004)

8. Rowstron, A.I.T., Druschel, P.: Pastry: Scalable, Decentralized Object Location, and Routing for Large-Scale Peer-to-Peer Systems. In: Guerraoui, R. (ed.) Middleware 2001. LNCS, vol. 2218, pp. 329-350. Springer, Heidelberg (2001)

9. Emulab, http://www.emulab.net

10. Brewer, E.: Towards Robust Distributed Systems (Invited Talk). In: Proc. PODC (2000) 
11. Jain, N., Kit, D., Mahajan, P., Yalagandula, P., Dahlin, M., Zhang, Y.: STAR: Self Tuning Aggregation for Scalable Monitoring. In: Proc. VLDB (2007)

12. Jain, N., Kit, D., Mahajan, P., Yalagandula, P., Dahlin, M., Zhang, Y.: PRISM: PrecisionIntegrated Scalable Monitoring (extended). In: Proc. OSDI (2008)

13. Park, K., Pai, V.S.: CoMon: a Mostly-scalable Monitoring System for PlanetLab. SIGOPS OSR 40(1), 65-74 (2006)

14. Massie, M.L., Chun, B.N., Culler, D.E.: The Ganglia Distributed Monitoring System: Design, Implementation and Experience. Parallel Computing 30(7) (2004)

15. PlanetLab: Contributed Software, https://wiki.planet-lab.org/twiki/bin/ view/Planetlab/ContributedSoftware

16. Zhao, B.Y., Huang, L., Stribling, J., Rhea, S.C., Joseph, A.D., Kubiatowicz, J.: Tapestry: A Resilient Global-scale Overlay for Service Deployment. IEEE JSAC 22(1) (2004)

17. Stoica, I., Morris, R., Karger, D., Kaashoek, F., Balakrishnan, H.: Chord: A Scalable Peerto-Peer Lookup Service for Internet Applications. In: Proc. SIGCOMM (2001)

18. Castro, M., Druschel, P., Kermarrec, A.M., Rowstron, A.: SCRIBE: A Large-scale and Decentralised Application-level Multicast Infrastructure. IEEE JSAC (2002)

19. Castro, M., Druschel, P., Kermarrec, A.M., Nandi, A., Rowstron, A., Singh, A.: SplitStream: High-bandwidth Multicast in a Cooperative Environment. In: Proc. SOSP (2003)

20. Ko, S.Y., Yalagandula, P., Gupta, I., Talwar, V., Milojicic, D., Iyer, S.: Moara: Flexible and Scalable Group-Based Aggregation System. Technical Report UIUCDCS-R-2008-2989, UIUC (2008)

21. Huebsch, R., Chun, B., Hellerstein, J.M., Loo, B.T., Maniatis, P., Roscoe, T., Shenker, S., Stoica, I., Yumerefendi, A.R.: The Architecture of PIER: an Internet-Scale Query Processor. In: Proc. CIDR (2005)

22. Huebsch, R., Garofalakis, M., Hellerstein, J.M., Stoica, I.: Sharing Aggregate Computation for Distributed Queries. In: Proc. SIGMOD (2007)

23. Liang, J., Ko, S.Y., Gupta, I., Nahrstedt, K.: MON: On-demand Overlays for Distributed System Management. In: Proc. USENIX WORLDS (2005)

24. Nandi, A., Ganjam, A., Druschel, P., Ng, T.S.E., Stoica, I., Zhang, H., Bhattachargee, B.: SAAR: A Shared Control Plane for Overlay Multicast. In: Proc. NSDI (2007)

25. Bozdog, A., van Renesse, R., Dumitriu, D.: SelectCast: A scalable and self-repairing multicast overlay routing facility. In: Proc. SSRS (2003)

26. Roussopoulos, M., Baker, M.: CUP: Controlled Update Propagation in Peer-to-Peer Networks. In: USENIX (2003)

27. Yalagandula, P., Dahlin, M.: Shruti: A Self-Tuning Hierarchical Aggregation System. In: SASO (2007) 